\title{
Knowledge Structures Based Adaptive Testing Model
}

\author{
Xia $\mathrm{Li}^{1}$, Hao Zhang ${ }^{2}$, Huali Yang ${ }^{2}$, Guixin Xing ${ }^{2}$ \\ ${ }^{1}$ School of Media \& Communication, Wuhan Textile University, Hubei, 430073, Wuhan, P.R.China \\ ${ }^{2}$ National Engineering Research Center for E-learning, Central China Normal University, Wuhan 430079, China
}

\begin{abstract}
How to know the students' overall cognitive situation quickly and accurately by testing has been a hot issue in teaching. Based on the relevant research at home and abroad over the adaptive testing, and in view of the current adaptive testing is not intelligent enough, this paper presents a personalized intelligent selection method which combines the traditional dichotomy and knowledge state boundary method. In test error correction process, this paper proposed the recent knowledge state compatible set theory, by using this theory, this paper tries to use probabilistic approach to rationalize the irrational state of knowledge to achieve the maximum "real" of the level of output. Examples of verification found that the proposed adaptive test model could quickly and accurately measure the true level of students' cognitive structure, and greatly improve test efficiency.
\end{abstract}

Keywords—knowledge structure; adaptive test; intelligent selection; state of knowledge; error correction

\section{INTRODUCTION}

In the United States, "TOEFL", "GRE”, Cisco and Microsoft certification exams and other international exams have carried out on-line test in a large scale. McGraw - Hill Company's ALEKS system is a typical representative of the online test system, it shows computer application in knowledge space theory, and it provides good individual teaching environment and learning content [1]. RATH model is an adaptive hypertext mentoring model [2] based on network environment. In recent years, due to the potential advantages of the theory, skills measurement and the ability, the research trend has extend to the knowledge based behavior theory.

Based on the analysis of knowledge structure organization method during test process, this paper made a deep study for the selection method at the beginning of test, the selection strategies during test and error control after test. We proposed adaptive test model based on knowledge structure, and developed a sample system for verifying the correctness of the test model. In Section 2, the paper introduces the relevant domestic and foreign researchers who has conducted related research on KST. In Section 3 details of the adaptive test model proposed will be explained and in this part we designed the system to validate the proposed model, and finally make a summary of the paper.

This study was funded by the Humanity and Social Science Youth foundation of Ministry of Education (No. 10YJC870018), the National Office for Education Sciences Planning Project (grant number CCA140152), the National Programs for Science and Technology Development(grant number 2015BAK07B03), and the Fundamental Research Funds for the Central Universities(grant number CCNU15GF001).

\section{RELATED WORKS}

Classical test theory uses simple one-dimensional linear model with weak assumptions and large inaccuracy, but because of its complete theoretical system and it is easy to realize, it is still the basement for standardized tests [3]. Generalizability Theory (GT) starts from the range of the macro, inherits the random sample theory test ideas and regards validity of the test as the theoretical research priorities [4]. The basic idea is to measure from the real measure situation and propose the concept of different reliability coefficients, to design methods to identify sources of error and analyze and classify the reasons of error [5]. Since the GT uses a "parallel sampling immediately" model, it has some shortcomings, for instance, the sampling date analysis cannot be guaranteed. Meanwhile, GT carries out the error test depend on the components, which leads to the difficulty in controlling variance components [6].

To overcome the lack of classical theory and GT test, many researchers proposed Item Response Theory, a test theory starts from questions. Lord proposed IRT and computing method for the required parameters, making IRT a popular theory [7]. IRT is the theoretical basement for adaptive testing, and it provides different test contents for different ability levels of students. In some Western countries, item response theory is widely used in real life, network test has been widely used in the field of educational measurement, occupation measurement, and personnel measurement[9].

The 1990s Falmagne proposed knowledge space theory [9], which is a mathematical psychology model. Knowledge Space Theory define knowledge from different granularity. It organize questions in a space, each element within the space combines a number of issues, which represents the people's mastery of knowledge state, called the state of knowledge[10]. Dietrich Albert combines knowledge space theory and formal hypertext model, and skill function, and build a framework for intelligent tutoring system, in order to provide students a personalized way of learning [11].

Although the adaptive test based on the knowledge space theory has unparalleled prospects and advantages, it has drawbacks, mainly in the following areas:

- First, the existing knowledge structure relationship of the adaptive testing will become too complicated to build and manage as the number of test increases.

- Second, the current universal "dichotomy" selection strategies cannot be completely intelligent. So adaptive selection strategies need to be further improved in 
order to better reflect the characteristics of the students themselves.

- Third, in the course of the test, it did not rule out errors in the test. So error knowledge statistics will be included in the test result.

To solve these problems, we proposed an adaptive test model based on knowledge structure, We fully considered the selection method for initial of questions, the selection strategies during testing as well as the control of accuracy after the test. We also designed a self-designed adaptation of Cognitive Diagnosis algorithm, which give full consideration to the effects of speculation and mistakes on the test results, and ensure that the test model based on the knowledge structure can accurately measure students' cognitive status.

\section{ADAPTIVE TEST MODEL}

Adaptive test model mainly get users' capability diagnose by giving adaptive testing and evaluation of the user's ability level and cognitive deficits. This paper developed a comprehensive adaptive testing system based on adaptive test model. We improved selection strategy based on improved testing model to ensure the intelligence of the system and enhance the efficiency of test.

\section{A. Building of knowledge structure}

Building knowledge structure is a prerequisite for adaptive testing, and only after building reasonable knowledge structure can we conduct adaptive test accurately. We need to build knowledge structures according to the structure relationship between test contents, so as to get filter to extract the structure of the relationship between the questions, and then get the knowledge structure consistent with the rules, namely learning path.

All the knowledge should have been classified and stored in the database. During the test we will select knowledge point firstly, build domain knowledge tree, and consider the relationship between knowledge points contained in each question. The last step is to build relational structure of the questions. The whole process contains five steps, as is shown in Figure 1, the following details will descript these five steps:

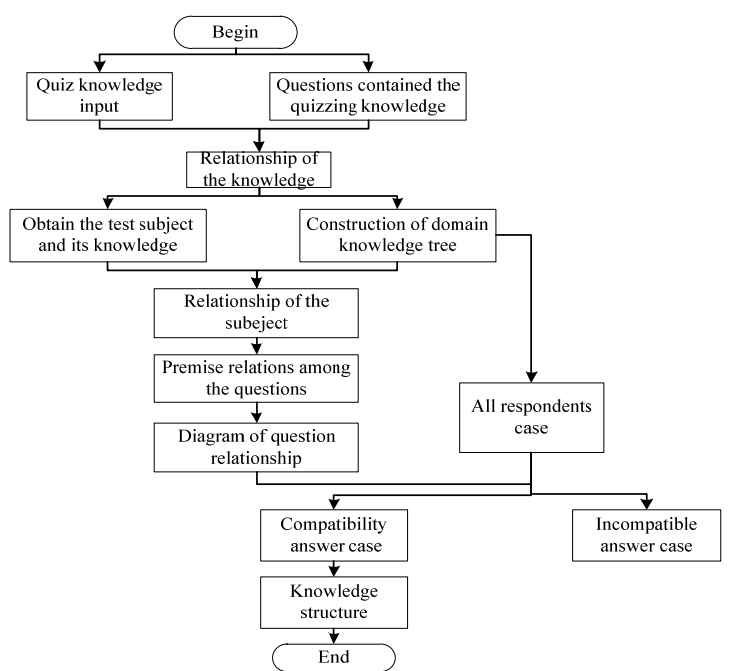

Figure 1. Flowchart for building the knowledge structure
Step one: Carding knowledge relationship, forming a hierarchical structure;

In order to construct knowledge tree, we need to obtain the knowledge points required in the test, classify them, identify the relationship between knowledge points, and build knowledge structure relation diagram according to test contents. The flow chart is shown is Figure 2.

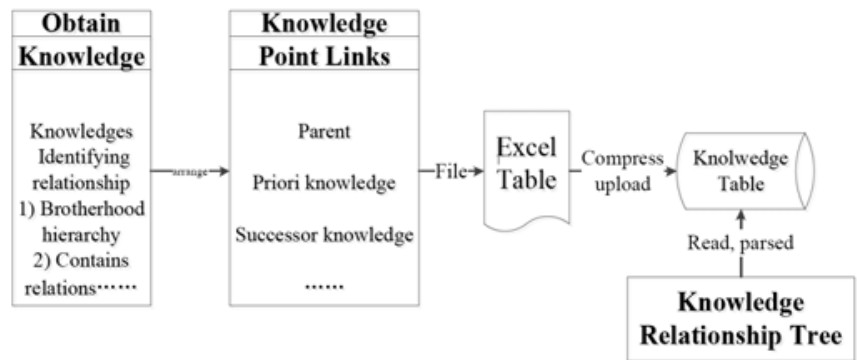

Figure 2. Construction and showing the relationship of the knowledge tree

Step two: Screening Title

Build test structure according to the given knowledge point, and gain the combination between questions.

Step three: Get relationship between questions

In order to obtain the relationship between the two questions, we need to conduct comparative judgment among knowledge points contained in each question.

Step Four: Build premise relations between questions

This article focuses on studying the relationship between "and" and “or". For independent relationship does no help to adaptive test, we will not take it into consideration.

Step Five: Building Knowledge Structure.

Since there are only two kinds of state: right or wrong of the answer to the question, the states of each question will constitute a sequence. Different students' answers will form a

plurality of different sequences. If there are n questions, there will be $2^{\mathrm{n}}$ kinds of answering sequences. In this paper, 0 stands for wrong answer, while 1 means right answer. For convenience, we can get the answer situation with a series of 0,1 which represent a binary sequence.

There are some constraints (AND, OR, independent), not all of the answer is reasonable, so we need select and classify the structural relationship of questions, put the reasonable ones into one group, and the rest of it will be regarded as incompatible answers.

\section{B. Smart Question Selection Strategies}

Selection Strategies has been a key point of adaptive testing, but also a difficult point of testing, which determines the degree of adaption. After presenting initial questions to students, the system will automatically select nest question, which can test students' ability levels and cognitive structure according to how students answer the initial one. Therefore, the selection of next question plays an important role in the test and it directly affect the efficiency. In current research 
work, dichotomy selection strategy is mainly used. But it is mechanic and monotonous, and we need to calculate many times and then get the minimum question qi. Therefore, the system using the double screening strategy combined by current knowledge status and dichotomy, significantly reducing the number of test items, and truly realize personalization. The concrete steps are shown in Figure 3

According to Figure 3, it shows that intelligent question selection process is divided into three steps. Firstly, get the boundary for known knowledge state. Secondly, use dichotomy method to conduct secondary selection. Third, present the questions.

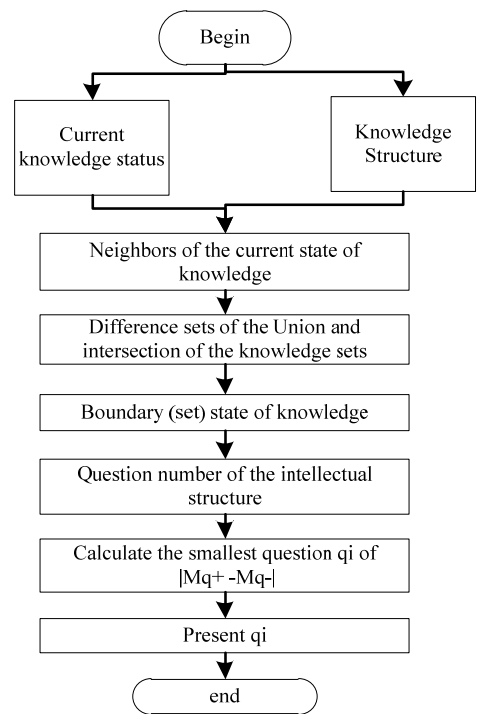

Figure 3. Smart Question Selection Strategies

\section{Correct the test error}

Error is quite a common thing in test, the error comes mainly from the students themselves in the proposed adaptive test method. For example, students may give wrong answers because of some certain factors, or they may failed in the question by guessing. These factors will lead to the error of selecting follow-up questions and make the overall test path inaccurate [12]. In this paper, we transfer the incompatible state of knowledge obtained from the test to the nearest compatible state [13], and calculate the error during the test process. It is shown in figure 5.

\section{Maximum close probability}

After the end of the test, the system needs to determine whether the answer is reasonable or not, for the irrational answer sequence, it requires to calculate their error. In this paper, we applied the probability transferring method to transfer the test results to the closest compatible answer state, and then present the error test to students.

Compare the knowledge state sequence results from test with reasonable one, we identify and count the variation of $0-1$ or 1-0 on each component, and record the number of component variation. Because the probability of failure or guessing is less than 1 , we only need to get the compatible answering state with minimal number of variation component. By comparison, variation minimal number of components to be compatible with the answer state, by contrast, the recently status set will be consist by the minimal variation of answer status.

\section{Probability calculating}

By contrast and judgment, we can get the recently compatibility set series of the test sequence, so we have to carry out the second judgment, if it only contains a sequence, the system can directly output. If it contains more than one, you will have to calculate the probability of every statue in test sequence, and compare and record the maximum probability of a compatible state sequence.

To facilitate calculation of the probability, we made the following definition of the questions:

Event A: test subject know one or more combinations of knowledge of the test question.

Event $\bar{A}$ : test subject did not grasp the combination of knowledge;

Event B: test subject answered the questions correctly;

Event $\bar{B}$ : test subject got the wrong answer of the questions;

Conditional probability $P(B \mid \bar{A})$ : the probability of guessing the test questions correctly.

Conditional probability $P(B \mid \bar{A})$ : the probability of answering the test questions incorrectly in the premise of knowing multiple (including one) knowledge point combinations of the test.; The probability of failure is not exactly a pre-assessment, it can only estimate by empirical data. In this paper, we evaluate the test subject by considering his habits, degrees of sloppy that shown in the course of test.

We calculate the guess probability and failure probability. According to the calculation method of total probability and Bayesian probability [14], we can get the following formula of guess probability and failure probability:

(1) the probability of guessing: When the test subject answer the test questions, he may not actually know any knowledge point combination. If we regard question as q, the probability of guessing is $\mathrm{Pg}(\mathrm{q})$. We can use the following formula to calculate it:

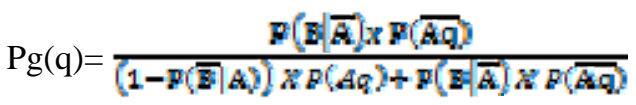

(2) The failure probability: When the test subject get wrong answer of test questions, he may know one or more than one knowledge point of the combination. If we regard the test question as q, the probability failure probability can be calculated according to the following formula:

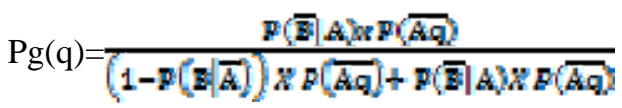


According to the above formula, we need to know the probability value of $P(A q)$ and $P(\overline{A q})$, because $P(A q)$ with $P(\overline{A q})$ is 1 , we just need to get $P(A q)$, then can get the result of $P(\overline{A q})$.

$\mathrm{P}(\mathrm{Aq})$ is calculated as follows: If the number of the tested knowledge point of question $\mathrm{q}$ is size (q), and there are two possibilities of each situation. So the possibilities of answering the test question is 2 size (q), and P (Aq) is the probability of situation that when the knowledge of test sequence is the same with the question sequence.

After the above analysis, we can infer the probability calculations, if one subject get $\mathrm{k} \mathrm{0-1}$ mistakes, i.e. guess $\mathrm{k}$ questions correctly; then we can calculate the knowledge status probability of the student and the compatibility status according to formula 3 :

$$
\prod_{k=1}^{k} P g(\mathrm{q}) \prod_{m=1}^{m} P \in(\mathrm{q})
$$

Including: $P g(q)$ for the subject's guess probability.

$\mathrm{Pe}(\mathrm{q})$ for the subject's failure probability.

\section{SYSTEM VERIFICATION}

In order to verify the proposed adaptive test model, in this section an adaptive testing system has been designed and implemented. During the process of designing it, we regard student as the main part, and measure the real ability level, learning characteristics, knowledge states and cognitive defects of the student by using intelligent selection strategies. At the meantime, we give timely analysis, feedback and recommend guidelines to lead students to a reasonable study path, focus, and overcome difficulty or weakness, and enhance learning in an all-round way.

Based on the proposed adaptive test system, we analyzed the test process by using a lot of questions. The test feedback is shown in Figure 4.

Test Results Feedback

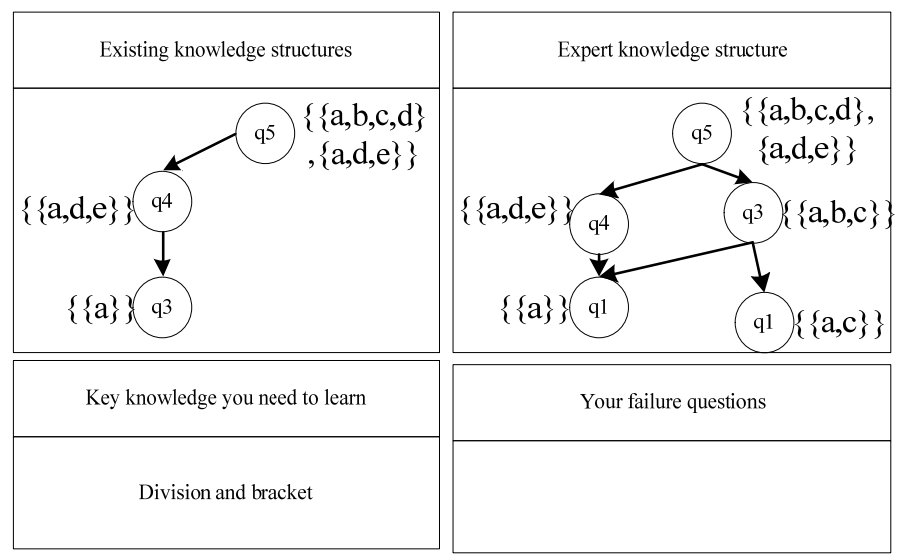

Figure 4. Test Result

\section{CONCLUSIONS}

This paper introduces adaptive test model, and then, explains the key points of adaptive test process in details. It can be divided into three parts, the method of constructing knowledge structure, the intelligent selection strategies, and the strategies for correcting errors after the test. The error correction process of transferring knowledge states has been proposed. Based on the propose adaptive testing model, we implemented an adaptive testing system. The real test has shown that this model can be used in adaptive testing, and to some extent, it reduce the errors of the test.

\section{References}

[1] Dowling, C. E, \& Hoekemeyer. C. Automata for the Assessment of Knowledge. IEEE Transactions on Knowledge and Data Engineering, 2001, 13, 451-461

[2] Maristella Agosti, Massimo Melucci, Fabio Crestani. Automatic authoring and construction of hypermedia for information retrieval [J]. Multimedia Systems. 1995 (1)

[3] Rong Xue. Two Mathematical Models of Language Testing: Response Theory from classical test theory to project [J]. Foreign Languages Research. 2007(04). (in Chinese)

[4] Zhiming Yang, Lei Zhang. Testing evaluation theory and its application [M]. Educational Science Press, 2003. (in Chinese)

[5] Brennan R L. Elements of Generalizability Theory.Iowa City:Act publications, 1983,35-41.

[6] Hambleton R K, Swaminathan H. Item response theory: Principles and applications. Boston: Kluwer Nijhoff Publishing, 1991, 201-206.

[7] Shuqing Qi. On the course of development of psychological and educational measurement theory. Journal of Jiangxi Normal University, 2001,34(1):94-99. (in Chinese)

[8] Zuwei $\mathrm{Xu}$, Item response theory and its application in tesing. Shanghai: East China Normal University Press,1992,30-34 (in Chinese)

[9] Falmagne J C, Koppen M, Villano M, et al. Introduction to knowledge spaces: How to build, test, and search them [J]. Psychological Review, 1990, 97(2): 201-224.

[10] Bin Zhu, Xianzhong Song. Dynamic web development technology [J]. Computer application. 2001(09) (in Chinese)

[11] Dietrich Albert, Cord Hockemeyer. Adaptive and Dynamic Hypertext Tutoring Systems Based on Knowledge Space Theory. International Journal of Man-Machine Studies, 520:555 2007

[12] Yanhua Liu. Research and Implementation of Adaptive Testing Method Based on Knowledge Space Theory [D]. Hunan University, 2010:16-33. (in Chinese)

[13] Yan Cheng. Theoretical Model of Adaptive Computer Test [J]. Computer and Modernization. 2006(09) (in Chinese).

[14] Jia-Sheng Heh, Shao-Chun Li, Alex Chang, Maiga Chang, Tzu-Chien Liu. Diagnosis Mechanism and Feedback System to Accomplish the Full-Loop Learning Architecture. Journal of Educational Technology \& Society. 2008 